\title{
Manifestaciones clínicas y radiológicas del derrame pleural en la UMAE No. 34 Monterrey, Nuevo León, México
}

\author{
Beatriz Mejía Olivares $\bowtie$
}

\author{
Servicio de Neumología, UMAE, Hospital de Cardiología No. 34, IMSS. Monterrey, Nuevo León, México.
}

Trabajo recibido: 22-X-2013; aceptado: 23-I-2014

\begin{abstract}
RESUMEN. Introducción: Muchas de las enfermedades benignas y malignas pueden causar un derrame pleural. Los síntomas dependen de la cantidad de líquido acumulado y la causa subyacente. Los estudios de imagen son útiles en la detección y el manejo de los derrames pleurales. Objetivos: Determinar las características radiológicas del derrame pleural y su relación con las manifestaciones clínicas, marcadores bioquímicos e histológicos. Material y métodos: De marzo de 2011 a marzo 2012 se realizó el estudio observacional, descriptivo, prolectivo y analítico. Se revisaron los estudios radiológicos de tórax de 138 pacientes mayores de 15 años de edad, con derrame pleural en estudio. Los estudios radiológicos fueron interpretados por tres neumólogos en forma ciega a la patología subyacente (kappa .95). Se utilizó el paquete estadístico SPSS 19.0. Se consideró significancia estocástica si el valor era $p<0.05$. Resultados: La edad media del grupo de estudio fue $56.1 \pm 15.8$ años, $93(67.4 \%)$ hombres. Los signos y síntomas más frecuentes independientemente de la etiología fueron: disnea 108 casos $(78.2 \%)(p=0.033)$, tos $96(69.5 \%)$, pérdida de peso $82(59.4 \%)(p=0.042)$ y dolor $79(57.2 \%)$. El patrón radiológico más común fue el derrame masivo 46 (33\%), seguida de los loculados 33 (24\%). Predominando los unilaterales 119 (86\%), con mayor frecuencia en los de etiología infecciosa $(p=0.012)$ y neoplásica $(p=0.006)$. Conclusiones: Las manifestaciones clínicas y radiológicas del derrame pleural son variadas, según el tipo de patología pleural y el grado de afectación de la misma.
\end{abstract}

Palabras clave: Derrame pleural, neoplasia, infecciosos, trasudados.

ABSTRACT. Introduction: Many benign and malignant diseases can cause pleural effusion. Symptoms depend on the amount of accumulated fluid and the underlying cause. Imaging studies are useful in the detection and management of pleural effusions. Objectives: To determine the radiological characteristics of pleural effusion and its relationship with clinical and biochemical manifestations and histological markers. Methods: From March 2011 to March 2012, an observational, descriptive, prolective analytical study was performed. Chest X-rays of 138 patients $>15$ years of age with pleural effusion during the study period were reviewed. Radiological studies were interpreted by three pulmonologists blinded to the underlying pathology (kappa .95). We used the statistical package SPSS 19.0. Statistical significance was considered when p value was < 0.05. Results: The mean age of the study group was $56.1 \pm 15.8$ years; $93(67.4 \%)$ were males. The most common signs and symptoms regardless of the etiology were dyspnea, 108 cases $(78.2 \%)(p=0.033)$; cough, $96(69.5 \%)$; weight loss, $82(59.4 \%)(p=0.042)$; and pain, $79(57.2 \%)$. The most common X-ray pattern was massive effusion in 46 (33\%) followed by loculated effusion in $33(24 \%)$. There was a unilateral predominance in $119(86 \%)$ patients, with greater frequency in those with infectious $(p=0.012)$ and neoplastic etiology $(p=$ 0.006). Conclusions: Clinical and radiological manifestations of pleural effusion are varied according to the type of pleural pathology and the degree of involvement.

Key words: Pleural effusion, neoplasm, infections, transudate.

\section{INTRODUCCIÓN}

Las enfermedades de la pleura se manifiestan habitualmente por el síndrome de ocupación pleural, que consiste en la acumulación de aire (neumotórax) o líquido (derrame pleural o pleuresía) en la cavidad pleural. El síndrome de ocupación pleural por líquido es, con gran diferencia, el más frecuente y variado, constituye hasta $10 \%$ de las patologías atendidas en los servicios de neumología..$^{1-3}$ Son un problema médico común, con más de 50 causas reconocidas incluyendo enfermedad local de la pleura o pulmón subyacente, condiciones sistémicas, disfunción de órganos y medicamentos. ${ }^{4}$

Los signos y síntomas varían dependiendo de la causa subyacente, pero disnea, tos y dolor pleurítico son los más comunes. La imagen radiográfica desempeña un papel importante en el diagnóstico y manejo de la enfermedad pleural. La radiografía de tórax es la técnica radiológica inicial en el estudio del derrame pleural, siendo la ecografía, la tomografía computarizada y la resonancia magnética técnicas complementarias en el abordaje posterior. ${ }^{5}$ 
Nuestro hospital es un centro de referencia del noreste del país, donde el derrame pleural es la segunda causa de ingreso hospitalario; razón por la que se describen las manifestaciones clínicas y radiológicas del derrame pleural y se valora su utilidad diagnóstica.

\section{MATERIAL Y MÉTODOS}

Estudio observacional, descriptivo, prolectivo y analítico.

Lugar de estudio: Servicio de Neumología del Hospital de Cardiología, Unidad Médica de Alta Especialidad (UMAE) No. 34, IMSS, Monterrey, Nuevo León, México, durante el período de marzo 2011 a marzo 2012. Las variables incluidas fueron las características demográficas, clínicas, bioquímicas e histológicas analizadas por las pruebas convencionales utilizando el paquete estadístico SPSS 19.0. Se consideró significancia estocástica si el valor era $\mathrm{p}<0.05$.

Población de estudio: Se estudiaron las características radiológicas de 138 pacientes consecutivos con diagnóstico de derrame pleural en estudio. Las características clínicas analizadas fueron: disnea, tos, pérdida de peso, expectoración, fiebre, hemoptisis y dolor tipo pleurítico (caracterizado por dolor en el costado que aumenta con la respiración, la tos, y con los movimientos del tórax, y puede desaparecer o minimizarse con la apnea). Los estudios radiológicos de tórax (Rx y TAC) fueron evaluados e interpretados por tres neumólogos en forma ciega a la patología subyacente, los casos de duda o controversia fueron resueltos por consenso, el índice de Kappa interobservador aplicado para concordancia de la imagen fue de 0.95 . El derrame pleural se clasificó en unilateral (derecho o izquierdo) o bilateral, por sus características radiográficas fueron clasificados en borramiento del ángulo costofrénico, subpulmonar, típicos (curva de Damaseiu o menisco), loculados, atípicos (cuando el pulmón presenta patología localizada y una elasticidad alterada pierde la capacidad de retracción, no conserva su forma y el líquido pleural puede adoptar formas atípicas) y masivos (el líquido ocupa $>2 / 3$ del hemitórax). El tamaño del derrame pleural en el hemitórax afectado se evaluó trazando dos líneas horizontales imaginarias, la primera se extendió desde la línea medioesternal hasta la axilar anterior, a la altura del tercer cartílago costal; y la segunda fue paralela a la anterior a nivel del sexto cartílago costal, dividiendo al hemitórax en tres tercios. En el caso de los derrames bilaterales se evaluó el lado afectado de mayor tamaño, en caso de existir afección parenquimatosa y mediastinal se clasificó con base en los patrones radiológicos de Fleischner Society: Glossary of Terms for Thoracic Imaging. ${ }^{6}$
El diagnóstico etiológico se estableció con base en las características bioquímicas, reacción en cadena de polimerasa (PCR), cultivos y citología del líquido y/o biopsia pleural. Se eligió a los pacientes mediante una técnica de muestreo no probabilístico de casos consecutivos.

\section{RESULTADOS}

Durante el período de marzo 2011 a marzo 2012 ingresaron al Servicio de Neumología 138 pacientes con diagnóstico de derrame pleural en estudio. Se estudiaron 45 mujeres (32.6\%) y 93 hombres $(67.4 \%)$. La edad media fue de $56.1 \pm 15.8$ años.

De las manifestaciones clínicas referidas por los pacientes, la más común fue la disnea en 108 pacientes (78.3\%); siendo el grado 3 de disnea de la escala modificada MRC (del inglés medical research council) la más frecuente con 48 casos (34.8\%); seguido del grado 4 con 45 casos (32.6\%). La tos se presentó en 96 pacientes, (69.6\%); pérdida de peso en 82 pacientes, (59.4\%); y el dolor en 79 pacientes, (57.2\%). La sintomatología fue muy similar en el grupo de pacientes $(p>0.05)$. En la tabla 1 se esquematiza el cuadro clínico referido por nuestra población.

En cuanto a las manifestaciones radiológicas de los 138 derrames pleurales, el tipo de derrame pleural predominante fue el masivo con 46 casos (33.3\%), seguido de los derrames loculados con 33 casos (23.9\%), destacaron los derrames unilaterales en 119 casos (86.2\%), siendo el hemitórax derecho el más afectado con 62 casos (44.9\%). En lo referente al número de tercios mayormente afectados éste fue de $2 / 3$ en 54 casos $(39.2 \%)$ y de $3 / 3$ en 47 casos (34\%) (tabla 1 ).

En los hallazgos tomográficos sólo $31(22.4 \%)$ pacientes presentaron alteraciones mediastinales, siendo más frecuentes las adenopatías mediastinales en 27 casos (19.6\%). En el parénquima pulmonar los patrones radiológicos con mayor frecuencia encontrados fueron las masas, 21 casos (15.2\%); imagen en vidrio despulido, 12 casos (8.6\%); nódulos múltiples, 11 casos (8.0\%); y nódulos únicos, 6 casos (4.3\%) (tabla 1 ).

Con base en el diagnóstico etiológico confirmado por estudios bioquímicos, cultivos e histopatología del líquido y biopsia pleural, se decidió asociar las manifestaciones clínicas y radiológicas por etiología documentada y comparar resultados (tabla 2), encontrando que las manifestaciones clínicas y radiológicas son muy características de acuerdo a cada etiología, con resultados similares. En el grupo de los derrames paraneumónicos, empiemas, tuberculosis y trasudados, la pérdida de peso se presentó con mayor frecuencia $(p=0.016)$, y la disnea predominó en los derrames pa- 
Tabla 1. Manifestaciones clínicas y radiológicas del derrame pleural.

\begin{tabular}{|c|c|c|c|}
\hline \multicolumn{4}{|c|}{ Núm. de casos 138} \\
\hline Signos y síntomas & Frecuencia & Porcentaje & Valor de $p$ \\
\hline Disnea & 108 & 78.3 & 0.033 \\
\hline $\begin{array}{c}\text { Grado de disnea MRC modificada } \\
0 \\
1 \\
2 \\
3 \\
4 \\
\end{array}$ & $\begin{array}{r}30 \\
2 \\
13 \\
48 \\
45\end{array}$ & $\begin{array}{r}21.7 \\
1.4 \\
9.4 \\
34.8 \\
32.6\end{array}$ & \\
\hline Tos & 96 & 69.6 & \\
\hline Pérdida de peso & 82 & 59.4 & 0.042 \\
\hline $\begin{array}{l}\text { Dolor } \\
\text { Expectoración } \\
\text { Fiebre } \\
\text { Hemoptisis }\end{array}$ & $\begin{array}{l}79 \\
61 \\
52 \\
12\end{array}$ & $\begin{array}{r}57.2 \\
44.2 \\
37.7 \\
8.7\end{array}$ & \\
\hline \multicolumn{4}{|l|}{ Tipo de derrame } \\
\hline $\begin{array}{l}\text { Masivo } \\
\text { Loculado } \\
\text { Típico } \\
\text { Atípico } \\
\text { Borramiento ángulo costofrénico }\end{array}$ & $\begin{array}{r}46 \\
33 \\
29 \\
27 \\
3\end{array}$ & $\begin{array}{r}33.3 \\
23.9 \\
21 \\
19.6 \\
2.2\end{array}$ & \\
\hline \multicolumn{4}{|l|}{ Localización derrame } \\
\hline $\begin{array}{l}\text { Unilateral } \\
\text { Derecho } \\
\text { Izquierdo } \\
\text { Bilateral }\end{array}$ & \begin{tabular}{|l|}
119 \\
62 \\
57 \\
19
\end{tabular} & $\begin{array}{l}86.2 \\
44.9 \\
41.3 \\
13.8 \\
\end{array}$ & \\
\hline \multicolumn{4}{|l|}{ Tercios afectados derecho } \\
\hline $\begin{array}{l}1 \text { tercio } \\
2 \text { tercios } \\
3 \text { tercios }\end{array}$ & $\begin{array}{l}13 \\
27 \\
22\end{array}$ & $\begin{array}{r}9.4 \\
19.6 \\
15.9\end{array}$ & \\
\hline \multicolumn{4}{|l|}{ Tercios afectados izquierdo } \\
\hline $\begin{array}{l}1 \text { tercio } \\
2 \text { tercios } \\
3 \text { tercios }\end{array}$ & $\begin{array}{r}5 \\
27 \\
25\end{array}$ & $\begin{array}{r}3.6 \\
19.6 \\
18.1\end{array}$ & \\
\hline \multicolumn{4}{|l|}{$\begin{array}{l}\text { Estudios tomográficos } \\
\text { Lesiones mediastinales }\end{array}$} \\
\hline $\begin{array}{l}\text { Ninguna } \\
\text { Ensanchamiento mediastinal } \\
\text { Adenopatías mediastinales } \\
\text { Masa mediastinal }\end{array}$ & $\begin{array}{r}107 \\
2 \\
27 \\
2 \\
138\end{array}$ & $\begin{array}{r}77.5 \\
1.4 \\
19.6 \\
1.4 \\
100\end{array}$ & \\
\hline \multicolumn{4}{|l|}{ Imágenes acompañantes } \\
\hline $\begin{array}{l}\text { Masa } \\
\text { Vidrio despulido } \\
\text { Nódulos múltiples } \\
\text { Nódulo único } \\
\text { Patrón micronodular } \\
\text { Llenado alveolar unilobar } \\
\text { Cavernas } \\
\text { Patrón nodular } \\
\text { Patrón reticular } \\
\text { Patrón reticulonodular } \\
\text { Llenado alveolar multilobar } \\
\text { Bronquiectasias } \\
\text { Bulas }\end{array}$ & $\begin{array}{r}21 \\
12 \\
11 \\
6 \\
5 \\
4 \\
3 \\
1 \\
2 \\
2 \\
2 \\
1 \\
2\end{array}$ & $\begin{array}{c}15.2 \\
8.6 \\
8 \\
4.3 \\
3.6 \\
2.9 \\
2.2 \\
0.7 \\
1.4 \\
1.4 \\
1.4 \\
1.4 \\
1.4\end{array}$ & \\
\hline
\end{tabular}

La sintomatología más frecuente en el derrame pleural independientemente de la etiología fue: disnea, tos, pérdida de peso y dolor torácico. Por sus características radiológicas predominó el derrame pleural unilateral y con afectación del hemitórax derecho. 
Tabla 2. Manifestaciones clínicas y radiológicas del derrame pleural según etiología.

\begin{tabular}{|c|c|c|c|c|c|}
\hline $\begin{array}{l}\text { Características } \\
\text { clínicas }\end{array}$ & $\begin{array}{l}\text { Paraneumónicos y } \\
\text { empiemas } \\
\text { (Núm. 43) }\end{array}$ & $\begin{array}{l}\text { Tuberculosis } \\
\text { (Núm. 17) }\end{array}$ & $\begin{array}{l}\text { Micosis } \\
\text { (Núm. 3) }\end{array}$ & $\begin{array}{l}\text { Neoplasias } \\
\text { (Núm. 45) }\end{array}$ & $\begin{array}{l}\text { Trasudados } \\
\text { (Núm. 7) }\end{array}$ \\
\hline Disnea & $\begin{array}{l}26(60 \%) \\
p=0.046\end{array}$ & 15 (88\%) & $2(67 \%)$ & $41(91 \%)$ & $6(86 \%)$ \\
\hline Tos & $33(77 \%)$ & $15(88 \%)$ & $3(100 \%)$ & $27(60 \%)$ & $5(71 \%)$ \\
\hline Pérdida de peso & $\begin{array}{l}21(49 \%) \\
p=0.016\end{array}$ & $\begin{array}{l}13(76 \%) \\
p=0.016\end{array}$ & $2(67 \%)$ & $29(64 \%)$ & $\begin{array}{r}1(14 \%) \\
n=00 ? 2\end{array}$ \\
\hline Dolor & $27(63 \%)$ & $13(76 \%)$ & $2(67 \%)$ & $26(58 \%)$ & $0 \%$ \\
\hline Fiebre & $28(65 \%)$ & $10(59 \%)$ & $0 \%$ & $7(15 \%)$ & $0 \%$ \\
\hline Expectoración & $23(53 \%)$ & $10(59 \%)$ & $3(100 \%)$ & $13(29 \%)$ & $3(43 \%)$ \\
\hline Hemoptisis & $1(2.3 \%)$ & $1(6 \%)$ & $1(33 \%)$ & $7(15 \%)$ & $0 \%$ \\
\hline \multicolumn{6}{|l|}{$\begin{array}{l}\text { Características } \\
\text { radiológicas }\end{array}$} \\
\hline Típicos & $2(5 \%)$ & $5(29 \%)$ & $2(67 \%)$ & $10(22 \%)$ & $2(28 \%)$ \\
\hline Atípicos & $10(23 \%)$ & $4(23 \%)$ & $1(33 \%)$ & $7(15 \%)$ & $3(43 \%)$ \\
\hline Loculados & $22(51 \%)$ & $2(12 \%)$ & & $4(9 \%)$ & \\
\hline Masivos & $9(21 \%)$ & $5(29 \%)$ & & $22(49 \%)$ & $2(28 \%)$ \\
\hline $\begin{array}{l}\text { Borramiento del } \\
\text { ángulo costofrénico }\end{array}$ & & & & $2(4 \%)$ & \\
\hline \multirow[t]{2}{*}{ Unilateral } & $39(91 \%)$ & $15(88 \%)$ & $3(100 \%)$ & $41(91 \%)$ & \\
\hline & $p=0.012$ & $p=0.012$ & $p=0.012$ & $p=0.006$ & \\
\hline Bilateral & $4(9 \%)$ & $2(12 \%)$ & & $4(9 \%)$ & $5(71 \%)$ \\
\hline Derecho & $22(51 \%)$ & $8(47 \%)$ & $1(33 \%)$ & $17(38 \%)$ & \\
\hline Izquierdo & $17(40 \%)$ & $7(41 \%)$ & $2(67 \%)$ & $24(53 \%)$ & \\
\hline Un tercio & $11(25 \%)$ & $6(35 \%)$ & $3(100 \%)$ & & $5(71 \%)$ \\
\hline Dos tercios & $25(58 \%)$ & $7(41 \%)$ & & $15(33 \%)$ & $5(71 \%)$ \\
\hline Tres tercios & $11(25 \%)$ & $6(35 \%)$ & & $22(49 \%)$ & $2(28 \%)$ \\
\hline \multirow{2}{*}{\multicolumn{6}{|c|}{$\begin{array}{l}\text { Imágenes } \\
\text { tomográficas }\end{array}$}} \\
\hline & & & & & \\
\hline Adenopatías & $7(16 \%)$ & $1(6 \%)$ & $1(33 \%)$ & $11(24 \%)$ & $4(57 \%)$ \\
\hline \multicolumn{6}{|l|}{ mediastinales } \\
\hline Patrón reticular & & $1(6 \%)$ & & & \\
\hline Patrón micronodular & & $3(18 \%)$ & $1(33 \%)$ & & \\
\hline Patrón reticulonodular & & $2(12 \%)$ & $1(33 \%)$ & $1(2.2 \%)$ & \\
\hline Llenado alveolar & $2(5 \%)$ & $1(6 \%)$ & $1(33 \%)$ & $1(2.2 \%)$ & $1(14.2 \%)$ \\
\hline Nódulos únicos & $1(2.3 \%)$ & $1(6 \%)$ & $1(33 \%)$ & $2(4.4 \%)$ & \\
\hline Nódulos múltiples & $1(2.3 \%)$ & $1(6 \%)$ & & $7(15.5 \%)$ & \\
\hline Caverna & $1(2.3 \%)$ & $1(6 \%)$ & & $1(2.2 \%)$ & \\
\hline Masa & & & $1(33 \%)$ & $12(27 \%)$ & \\
\hline
\end{tabular}

Causas más frecuentes del derrame pleural y sus manifestaciones clínicas y radiológicas, destacando los derrames pleurales unilaterales en forma significativa en los derrames de etiología infecciosa y neoplásica.

renumónicos y empiemas $(p=0.046)$. En cuanto a las manifestaciones radiológicas, los derrames unilaterales correspondieron con mayor frecuencia a los derrames infecciosos $(p=0.012)$ y neoplásicos $(p=0.006)$.

Por último, en el presente estudio sólo en 23 casos $(16.7 \%)$ no se logró determinar la etiología del derrame pleural a pesar de la repetición de pruebas diagnósticas, otros por mejoría clínica, y otros por no aceptar un segundo internamiento para la repetición de métodos diagnósticos.

\section{DISCUSIÓN}

Al analizar nuestro estudio se observó que la sintomatología de un paciente con derrame pleural es variada, depende de la causa subyacente (pleural, pulmonar o extrapulmonar), el grado de inflamación de la pleura y el compromiso de la mecánica pulmonar. Los signos y síntomas son específicos del sistema respiratorio acompañados de síntomas generales inespecíficos. ${ }^{7}$ En nuestro estudio, las manifestaciones clínicas pre- 
dominantes fueron: disnea, $78 \%(p=0.033)$; tos, $70 \%$; pérdida de peso, $59 \%$ ( $p=0.042)$, y dolor pleurítico, $57 \%$; similar a lo reportado en la literatura. ${ }^{7}$ La pérdida de peso se asoció a trasudados, tuberculosis, derrames paraneumónicos y empiemas $(p=0.016)$, la disnea predominó en los derrames parenumónicos y empiemas $(p=0.046)$.

El tamaño del derrame pleural acota el espectro etiológico; ${ }^{8}$ Porcel et al., en España encontraron 70 casos $(9 \%)$ de derrames pleurales que ocupan más de dos tercios del hemitórax afectado, y $93(12 \%)$ derrames masivos (obliteración completa del hemitórax) cuya principal etiología por orden de frecuencia fue la neoplásica (55\%), seguida de derrames paraneumónicos complicados y empiemas (22\%) y tuberculosis (12\%). ${ }^{9}$ En nuestra población se presentaron 46 casos de derrames masivos (33.3\%), 22 (16\%) de ellos de origen neoplásico, 9 (6.5\%) empiemas y derrames paraneumónicos, 5 (3.6\%) de tipo tuberculosis y $2(1.4 \%)$ derrames de tipo trasudado.

Los derrames loculados ocurren más comúnmente en asociación con condiciones que causan intensa inflamación pleural como el empiema, el hemotórax o la tuberculosis. ${ }^{8-12}$ En nuestro estudio los derrames loculados se presentaron en 33 casos (23.9\%), 22 (16\%) de ellos correspondieron a derrames paraneumónicos y empiemas, $4(3 \%)$ de origen neoplásico y $2(1.4 \%)$ de tipo tuberculosis.

Leung et al., en una revisión de estudios tomográficos de pacientes con enfermedad pleural encontraron que el hemitórax con mayor afectación fue el derecho con $41(57.7 \%)$ casos y el izquierdo con $33(44.5 \%)$, las causas más frecuentes fueron los exudados de tipo neoplasias y procesos infecciosos. ${ }^{13,14}$ Por nuestra parte, encontramos 119 (86.2\%) derrames pleurales unilaterales, $62(44.9 \%)$ del hemitórax derecho y 57 (41.3\%) del hemitórax izquierdo; 98 (71\%) de tipo exudado: 41 $(29.7 \%)$ de origen neoplásico, $39(28.2 \%)$ derrames paraneumónicos y empiemas, $15(10.8 \%)$ de tipo tuberculosis y $3(2.1 \%)$ de origen micótico (dos secundarios a coccidiodomicosis pulmonar y pleural, y el tercero secundario a aspergiloma). Derrames unilaterales de tipo trasudado se documentaron 2 (1.4\%), 1 secundario a diálisis peritoneal y otro secundario a falla cardíaca. La literatura reporta como causas más frecuentes de derrame pleural unilateral de tipo trasudado a la insuficiencia ventricular izquierda, cirrosis hepática, hipoalbuminemia y diálisis peritoneal. ${ }^{15}$

El diagnóstico diferencial en el derrame pleural bilateral es más estrecho e incluye causas de derrame de tipo trasudado. En nuestro estudio se documentaron un total de 7 (5\%) derrames de tipo trasudado; 5 de ellos de forma bilateral: 2 secundarios a insuficiencia cardíaca congestiva, 2 a insuficiencia renal crónica en tratamiento sustitutivo con diálisis peritoneal y hemodiálisis y 1 por cirrosis hepática, causas similares a lo reportado en la literatura. ${ }^{15}$

Por el contrario, la bilateralidad sin cardiomegalia asociada se relaciona frecuentemente con neoplasia. ${ }^{8-15}$ Pérez et al., en una revisión de 50 derrames pleurales bilaterales sin diagnóstico de insuficiencia cardíaca congestiva reportaron que la causa más frecuente la constituyó el cáncer, se consideró conjuntamente las metástasis de neoplasias pulmonares, extrapulmonares y las neoplasias hematológicas, con un total de 28 pacientes (56\%). ${ }^{16}$ En nuestro estudio, de un total de 131 (94.9\%) derrames pleurales de tipo exudado obtenidos se documentaron $10(7.2 \%)$ derrames bilaterales: 4 $(2.8 \%)$ de origen neoplásico, $4(2.8 \%)$ derrames paraneumónicos y empiemas, y $2(1.4 \%)$ de tipo tuberculosis. El empiema normalmente es unilateral, cuando es bilateral el volumen es mayor en el lado afectado.17,18

Las masas pulmonares, adenopatías mediastinales y la presencia de nódulos únicos o múltiples se observaron con mayor frecuencia en el grupo de las neoplasias, similar a lo reportado en la literatura. ${ }^{19,20}$

\section{CONCLUSIONES}

Con base en los datos obtenidos de nuestra población de estudio concluimos que el cuadro clínico predominante del derrame pleural fue la disnea, tos, pérdida de peso y el dolor pleurítico, similar a lo reportado en la literatura.

Las manifestaciones radiológicas que el derrame pleural adopta son diversas, observando un mayor número de derrames pleurales unilaterales en los derrames de etiología infecciosa y neoplásica $(<0.012)$, y los derrames pleurales de tipo trasudado fueron de predominio bilateral.

\section{REFERENCIAS}

1. Villena GV, Ferrer SJ, Hernández BL, et al. Diagnóstico y tratamiento del derrame pleural. Arch Bronconeumol 2006;42(7):349-372.

2. Andreu SJ, Arce GJ. Pleura. AMF 2007;3(10):556-559.

3. Pérez RE, Villena GMA, editores. Enfermedades de la pleura. Monografías NEUMOMADRID. Madrid: Ergon; 2003.

4. Hooper C, Lee YC, Maskell N; BTS, Pleural guideline Group. Investigation of a unilateral pleural effusion in adults: British Thoracic Society Pleural Disease Guideline 2010. Thorax 2010;65 Suppl 2:ii4-ii17.

5. Pedrosa CS. Diagnóstico por imagen. Tórax. 3ra ed. Madrid: Marban 2009;3:365-376. 
6. Hansell DM, Bankier AA, MacMahon H, McLoud TC, Müller NL, Remy J. Fleischner Society: glossary of terms for thoracic imaging. Radiology 2008;246(3):697-722.

7. Cicero SR, Páramo ARF, Navarro RFP, Pimentel UL. Procedimientos quirúrgicos en 156 casos de derrame pleural. Resultados inmediatos. Rev Cir Ciruj 2006;74(6):409-414.

8. Porcel JM, Caballero MR. Evaluación diagnóstica del derrame pleural. Med Clin (Barc) 2004;123(11):426-432.

9. Porcel JM, Vives M. Etiology and pleural fluid characteristics of large and massive effusions. Chest 2003;124(3):978-983.

10. Guía de referencia rápida: diagnóstico y tratamiento del derrame pleural. México: Secretaría de Salud; 2009.p.1-9.

11. Páez PI, Pino APP, Gassiot NC, Rodríguez VJC, Placeres FA. Derrame pleural paraneumónico y empiema pleural. Acta Médica 2000;9(1-2):52-58.

12. Asensio O, Blanco J, Moreno A, Pérez J, Salcedo A, Sanz L. Manejo de los derrames pleurales paraneumónicos. An Esp Pediatr 2001;54(3):272-282.

13. Leung AN, Müller NL, Miller RR. CT in differential diagnosis of diffuse pleural disease. AJR Am J Roentgenol 1990;154(3):487-492.

14. Rodríguez-Panadero F, Aguilar PM, Muñoz MMA, Asencio CMI. Manejo de la patología pleural. Arch Bronconeumol 2009;45(Suppl 3):22-27.
15. McGrath EE, Anderson PB. Diagnosis of pleural effusion: a systematic approach. Am J Crit Care 2011;20(2):119127.

16. Pila Pérez R, Pila Peláez R, Holguín Prieto VA. Derrame pleural bilateral en pacientes sin insuficiencia cardíaca. AMC (revista en línea) 2010;14(2).

17. Müller NL. Imaging of the pleura. Radiology 1993;186(2):297-309.

18. Evans AL, Gleeson FV. Radiology in pleural disease: state of the art. Respirology 2004; 9(3):300-312.

19. Oropesa CA, Soto GS. Derrame pleural neoplásico. Cuad Cir 2002;16:92-99.

20. Porcel PJM. Pruebas diagnósticas. Estudio del derrame pleural. JANO (revista en línea) 2009;(1.755):25-29.

\section{$\triangle$ Correspondencia:}

Dra. Beatriz Mejía Olivares

UMF/HGZ No.1 IMSS. Calle Ixmiquilpan 213,

Colonia Céspedes, Pachuca, Hidalgo. 42090

Correo electrónico: betty290775@hotmail.com

El autor declara no tener conflictos de interés. 